Research Article

\title{
On the Hadamard Well-Posedness of Generalized Mixed Variational Inequalities in Banach Spaces
}

\author{
Lu-Chuan Ceng $\mathbb{D}^{1},{ }^{1}$ Yeong-Cheng Liou $\mathbb{D}^{2,3}$ Ching-Feng Wen ${ }^{2},{ }^{4}$ Hui-Ying Hu, ${ }^{1}$ \\ Long $\mathrm{He}^{1}$ and Yun-Ling Cui ${ }^{1}$ \\ ${ }^{1}$ Department of Mathematics, Shanghai Normal University, Shanghai 200234, China \\ ${ }^{2}$ Department of Healthcare Administration and Medical Informatics, Center for Big Data Analytics \& Intelligent Healthcare, \\ and Research Center of Nonlinear Analysis and Optimization, Kaohsiung Medical University, Kaohsiung 807, Taiwan \\ ${ }^{3}$ Department of Medical Research, Kaohsiung Medical University Hospital, Kaohsiung 80708, Taiwan \\ ${ }^{4}$ Center for Fundamental Science and Research Center for Nonlinear Analysis and Optimization, Kaohsiung Medical University, \\ Kaohsiung 80708, Taiwan
}

Correspondence should be addressed to Lu-Chuan Ceng; zenglc@shnu.edu.cn

Received 2 September 2021; Accepted 25 October 2021; Published 18 November 2021

Academic Editor: Yongqiang Fu

Copyright (c) $2021 \mathrm{Lu}$-Chuan Ceng et al. This is an open access article distributed under the Creative Commons Attribution License, which permits unrestricted use, distribution, and reproduction in any medium, provided the original work is properly cited.

We introduce a new concept of Hadamard well-posedness of a generalized mixed variational inequality in a Banach space. The relations between the Levitin-Polyak well-posedness and Hadamard well-posedness for a generalized mixed variational inequality are studied. The characterizations of Hadamard well-posedness for a generalized mixed variational inequality are established.

\section{Introduction}

In [1], Tykhonov first introduced the well-posedness of a minimization problem, which means that it has a unique minimizer and every minimizing sequence converges to the unique minimizer. There are two concepts of well-posedness which are Tykhonov well-posedness [1] and Hadamard wellposedness [2].

Recently, variational inequality (VI) has been extensively studied due to the facts that it has many potential applications and that it is closely related to a differentiable minimization problem. Well-posedness for a variational inequality has been then extensively investigated. See, e.g., [3-11] and the references therein.

In 2013, Li and Xia [12] introduced the concept of Hadamard well-posedness of a general mixed variational inequality in Banach spaces. Under some suitable conditions, relations between Levitin-Polyak well-posedness and Hadamard well-posedness of a general mixed variational inequality were presented. They also established some characterizations of Hadamard well-posedness for a general mixed variational inequality. Very recently, some scholars still focused on the study of the well-posedness of various classes of variational inequalities, see e.g., generalized variational-hemivariational inequalities with perturbations in [13], completely generalized mixed variational inequalities in [14], noncompact generalized mixed variational inequalities in [15], generalized variational inequality with generalized mixed variational inequality constraint in [16], systems of generalized mixed quasivariational inclusion problems in [17], systems of time-dependent hemivariational inequalities in [18], and generalized hemivariational inequalities in [19].

Motivated and inspired by the research work going on this field, we introduce a new concept of Hadamard wellposedness for a generalized mixed variational inequality in a Banach space. Under some suitable conditions, the relations between the Levitin-Polyak well-posedness and Hadamard well-posedness for a generalized mixed variational inequality are studied. We also establish some 
characterizations of Hadamard well-posedness for a generalized mixed variational inequality. Finally, we prove that under suitable conditions, the Hadamard well-posedness of a generalized mixed variational inequality is equivalent to the existence and uniqueness of its solutions. Our results improve, extend, and develop the earlier and recent ones announced by some others, e.g., Ceng and Yao [7] and Li and Xia $[12,20]$.

\section{Preliminaries}

Let $X$ be a real reflexive Banach space with its dual $X^{*}$ and $K$ be a nonempty, closed subset of $X$. We use the same notations in [12]. For more details about these notations and relevant definitions, please consult relevant reference; see, e.g., [12] (following [21]). Let $X^{\prime}$ be the collection of all affine functions defined on $X$. It is obvious that $X^{*} \subset X^{\prime}$. Let $U$ be the collection of all nonempty set-valued mappings $F: X \longrightarrow 2^{X^{*}}$, and $\tau(X)$ be the collection of all mappings $P: X \longrightarrow 2^{X^{\prime}}$ such that for any $x \in X$, there exist $F \in U$ and $\lambda \in \mathbf{R}$ such that

$$
\langle P(x), x-y\rangle=\langle F(x), x-y\rangle+\lambda, \quad \forall y \in K .
$$

For any $P_{1}, P_{2} \in \tau(X)$, it follows that there exist $F_{i} \in U$ and $\lambda_{i} \in \mathbf{R}, i=1,2$ such that

$$
\begin{array}{ll}
\left\langle P_{1}(x), x-y\right\rangle=\left\langle F_{1}(x), x-y\right\rangle+\lambda_{1}, & \forall y \in K, \\
\left\langle P_{2}(x), x-y\right\rangle=\left\langle F_{2}(x), x-y\right\rangle+\lambda_{2}, & \forall y \in K .
\end{array}
$$

We define

$$
d\left(P_{1}, P_{2}\right)= \begin{cases}\left|\lambda_{1}-\lambda_{2}\right|, & F_{1}=F_{2}, \\ 1+\left|\lambda_{1}-\lambda_{2}\right|, & F_{1} \neq F_{2} .\end{cases}
$$

It can be routinely checked that $(\tau(X), d)$ is a metric space. In particular, if $U$ is the collection of all single-valued mappings $F: X \longrightarrow X^{*}$ and $\tau(X)$ is the collection of all single-valued mappings $P: X \longrightarrow X^{\prime}$ such that for any $x \in X$, there exist $F \in U$ and $\lambda \in \mathbf{R}$ such that

$$
\langle P(x), x-y\rangle=\langle F(x), x-y\rangle+\lambda, \quad \forall y \in K,
$$

then the above metric space $(\tau(X), d)$ reduces to the metric space $(\tau(X), d)$ defined in [[12], p. 1619]. In this case, it is clear that the metric space $(\tau(X), d)$ is a special case of metric space $(\Gamma, d)$ defined in [[21], p. 377].

Let $C(X)$ be the collection of all nonempty closed subsets of $X$ endowed with the usual Hausdorff metric $\mathscr{H}(\cdot, \cdot)$, that is, for every $A_{1}, A_{2} \in C(X)$,

$$
\mathscr{H}\left(A_{1}, A_{2}\right)=\max \left\{e\left(A_{1}, A_{2}\right), e\left(A_{2}, A_{1}\right)\right\},
$$

where $\quad e\left(A_{1}, A_{2}\right)=\sup _{a \in A_{1}} d^{\prime}\left(a, A_{2}\right) \quad$ with $\quad d^{\prime}\left(a, A_{2}\right)$ $=\inf _{b \in A_{2}}\|a-b\|$. Let $\left\{A_{n}\right\}$ be a sequence of nonempty closed subsets of $X$. We say that $A_{n}$ converges to $A$ in the Hausdorff metric iff $\mathscr{H}\left(A_{n}, A\right) \longrightarrow 0$ as $n \longrightarrow \infty$.

Let $B(X)$ be the family of all real-valued functions on $X$; we define

$$
d_{1}\left(\phi_{1}, \phi_{2}\right)=\sup _{x \in X}\left|\phi_{1}(x)-\phi_{2}(x)\right|
$$

where $\phi_{1}, \phi_{2} \in B(X)$; it can be routinely checked that $\left(B(X), d_{1}\right)$ is a metric space.

Let $M$ be the collection of all $(P, \phi, K)$ such that

(i) $P \in \tau(X)$;

(ii) $\phi \in B(X)$;

(iii) $K \in C(X)$.

Then, for any $\left(P_{1}, \phi_{1}, K_{1}\right),\left(P_{2}, \phi_{2}, K_{2}\right) \in M$, we define $\rho\left(\left(P_{1}, \phi_{1}, K_{1}\right),\left(P_{2}, \phi_{2}, K_{2}\right)\right)=d\left(P_{1}, P_{2}\right)+d_{1}\left(\phi_{1}, \phi_{2}\right)+\mathscr{H}\left(K_{1}, K_{2}\right)$.

Clearly, $(M, \rho)$ is a metric space.

Let $F: X \longrightarrow 2^{X^{*}}$ be a set-valued mapping, and $\phi: X \longrightarrow \mathbf{R} \cup\{+\infty\}$ be a proper, convex, and lower semicontinuous functional. Consider the following generalized mixed variational inequality associated with $(F, \phi, K)$ :

$\operatorname{GMVI}(F, \phi, K)$ : find $x \in K$ such that for some $u \in F(x)$,

$$
\langle u, x-y\rangle+\phi(x)-\phi(y) \leq 0, \quad \forall y \in K .
$$

We denote by $S(F, \phi, K)$ the solution set of $\operatorname{GMVI}(F, \phi, K)$. In what follows, we first introduce new concept of Hadamard well-posedness for $\operatorname{GMVI}(F, \phi, K)$. It is worth mentioning that some similar ideas have also been presented in $[22,23]$ very recently.

Definition 1. A generalized mixed variational inequality $\operatorname{GMVI}(F, \phi, K)$ is called Hadamard well-posed if it has a unique solution $x^{*} \in K$, and if for every sequence of triples $\left\{\left(P_{n}, \phi_{n}, K_{n}\right)\right\} \subset M$ converging to $(F, \phi, K)$ and every sequence $\left\{x_{n}\right\}$ such that $x_{n} \in S\left(P_{n}, \phi_{n}, K_{n}\right)$ for each $n \in \mathbf{N}$, it follows that $x_{n} \longrightarrow x^{*}$, where $K \cup\left\{x_{n}\right\} \subseteq K_{n}$ for all $n \in \mathbf{N}$.

Definition 2 (see [20]). A sequence $\left\{x_{n}\right\} \subset X$ is called a LP approximating sequence for $\operatorname{GMVI}(F, \phi, K)$, if there exist $w_{n} \in X$ with $w_{n} \longrightarrow 0$ and $0<\epsilon_{n} \longrightarrow 0$ such that $x_{n}+w_{n} \in K$ for all $n \in \mathbf{N}$, and there exists $u_{n} \in F\left(x_{n}\right)$ such that

$$
\left\langle u_{n}, x_{n}-y\right\rangle+\phi\left(x_{n}\right)-\phi(y) \leq \epsilon_{n}, \quad \forall y \in K, n \in N .
$$

Definition 3 (see [20]). We say that $\operatorname{GMVI}(F, \phi, K)$ is LP well-posed if $\operatorname{GMVI}(F, \phi, K)$ has a unique solution and every LP approximating sequence for $\operatorname{GMVI}(F, \phi, K)$ converges strongly to the unique solution.

The product space $C(X) \times B(X)$ is equipped by a product metric, that is, $\mathscr{H}\left(A_{1}, A_{2}\right)+d_{1}\left(f_{1}, f_{2}\right)$, where $A_{1}, A_{2} \in C(X)$ and $f_{1}, f_{2} \in B(X)$. Let further $B C^{0}(X)$ be the family of all real-valued continuous functions on $X$; it is easy to check that $\left(B C^{0}(X), d_{1}\right)$ is a metric space and we write $Q=C(X) \times B C^{0}(X)$. Now, we can easily get the following lemma.

Lemma 1 (see [12]). Let the pair $(A, f) \in Q$, then the function $(A, f) \longmapsto \inf (A, f)$ is upper semicontinuous. 
We consider the following gap function for $\operatorname{GMVI}(F, \phi, K)$ :

$$
g(x)=\sup _{y \in K} \inf _{u \in F(x)}\{\langle u, x-y\rangle+\phi(x)-\phi(y)\}, \quad \forall x \in X .
$$

Lemma 2. The following statements hold:

(i) $g(x) \geq 0, \forall x \in K$;

(ii) $\bar{x} \in K$ solves $\operatorname{GMVI}(F, \phi, K) \Longleftrightarrow g(\bar{x})=0$.

Proof. For each $x \in K$, we have

$$
\begin{aligned}
g(x) & =\sup _{y \in K} \inf _{u \in F(x)}\{\langle u, x-y\rangle+\phi(x)-\phi(y)\} \\
& \geq \inf _{u \in F(x)}\{\langle u, x-x\rangle+\phi(x)-\phi(x)\} \\
& =0 .
\end{aligned}
$$

Observe that

$$
\begin{aligned}
& \bar{x} \text { solves } \operatorname{GMVI}(F, \phi, K) \\
& \Longleftrightarrow \exists \bar{u} \in F(\bar{x}) \text { s.t. }\langle\bar{u}, \bar{x}-y\rangle+\phi(\bar{x})-\phi(y) \leq 0, \quad \forall y \in K \\
& \Longleftrightarrow \sup _{y \in K}\{\langle\bar{u}, \bar{x}-y\rangle+\phi(\bar{x})-\phi(y)\} \leq 0 \\
& \Longleftrightarrow 0 \leq g(\bar{x}) \leq \sup _{y \in K}\{\langle\bar{u}, \bar{x}-y\rangle+\phi(\bar{x})-\phi(y)\} \leq 0 \\
& \Longleftrightarrow g(\bar{x})=0 .
\end{aligned}
$$

This completes the proof.

We also consider the following optimization problem:

$$
\text { (OP): } \inf _{x \in K} g(x)
$$

with $g(x)$ defined by (10). Its optimal solution set will be denoted by $\arg \min (K, g)$ and the optimal value will be denoted by inf $(K, g)$, respectively.

The following Definitions $4-6$ can be found in [12]. However, Definition 7 is conventional.

Definition 4. A sequence $\left\{x_{n}\right\} \subset X$ is called an LP minimizing sequence for (OP) if there exists $u_{n} \in F\left(x_{n}\right)$ such that

$$
\begin{aligned}
g_{n}\left(x_{n}\right) & \longrightarrow \inf (K, g), \\
d^{\prime}\left(x_{n}, K\right) & \longrightarrow 0,
\end{aligned}
$$

where $g_{n}\left(x_{n}\right)=\sup _{y \in K \cup\left\{x_{n}\right\}}\left\{\left\langle u_{n}, x{ }_{n}-y\right\rangle+\phi\left(x_{n}\right)-\phi\right.$ $(y)\}, \forall n \in \mathbf{N}$.

Definition 5. We say that (OP) is LP well-posed if and only if (OP) has a unique solution and every LP minimizing sequence for (OP) converges strongly to the unique solution.

Definition 6. A nonempty-valued function $f: X \longrightarrow \mathbf{R}$ $\cup\{+\infty\}$ is said to be uniformly continuous, if for any $\epsilon>0$, there exists $\delta>0$ such that for all $x, y \in X$ with $\|x-y\|<\delta$, one has $|f(x)-f(y)|<\varepsilon$.

Definition 7. A nonempty set-valued mapping $F: X \longrightarrow 2^{X^{*}}$ is said to be monotone, if for all $x, y \in X, u \in F(x)$ and $v \in F(y)$,

$$
\langle u-v, x-y\rangle \geq 0 \text {. }
$$

Definition 8 (see [7]). Let $\mathscr{H}(\cdot, \cdot)$ be the Hausdorff metric on the collection $\mathrm{CB}(X)$ of all nonempty, closed, and bounded subsets of $X$, which is defined by $\mathscr{H}(A, B)=\max \{e$ $(A, B), e(B, A)\}$ for $A$ and $B$ in $\mathrm{CB}(X)$. A nonempty setvalued mapping $F: X \longrightarrow 2^{X^{*}}$ is said to be

(i) $\mathscr{H}$-hemicontinuous, if for any $x, y \in X$, the function $t \longmapsto \mathscr{H}(F(x+t(y-x)), F(x))$ from $[0,1]$ into $\mathbf{R}^{+}=[0,+\infty)$ is continuous at $0^{+}$;

(ii) $\mathscr{H}$-uniformly continuous, if for any $\epsilon>0$, there exists $\delta>0$ such that for all $x, y \in X$ with $\|x-y\|<\delta$, one has $\mathscr{H}(F(x), F(y))<\epsilon$.

The following proposition is a special case of Lemma 2.2 in [7].

Proposition 1 (see [20]). Let $K$ be a nonempty, closed, and convex subset of $X, F: X \longrightarrow 2^{X^{*}}$ be a nonempty compactvalued mapping which is $\mathscr{H}$-hemicontinuous and monotone, and $\phi: X \longrightarrow \mathbf{R} \cup\{+\infty\}$ be a proper and convex functional. Then, for a given $x \in K$, the following statements are equivalent:

(i) there exists $u \in F(x)$ such that $\langle u, x-y\rangle+\phi(x)$ $-\phi(y) \leq 0, \forall y \in K$

(ii) $\langle v, x-y\rangle+\phi(x)-\phi(y) \leq 0, \forall y \in K, v \in F(y)$.

We can also prove the following lemma easily.

Lemma 3. Let $K$ be a nonempty, closed subset of $X, F: X \longrightarrow 2^{X^{*}}$ be a nonempty set-valued mapping, and $\phi: X \longrightarrow \mathbf{R} \cup\{+\infty\}$ be a functional on $X$. Then, the following statements are equivalent:

(i) $\operatorname{GMVI}(F, \phi, K)$ is LP well-posed;

(ii) (OP) is LP well-posed with $g(x)$ defined by (10).

Proof. We first claim that (i) $\Rightarrow$ (ii). Indeed, suppose that $\operatorname{GMVI}(F, \phi, K)$ is LP well-posed and $x^{*} \in K$ is the unique solution of $\operatorname{GMVI}(F, \phi, K)$. By Lemma $2, x^{*} \in K$ is the unique solution of (OP). Then, we get $\inf (K, g)=0$. Let $\left\{x_{n}\right\} \subset X$ be a LP minimizing sequence for (OP). Then, there exists $u_{n} \in F\left(x_{n}\right)$ such that

$$
\begin{aligned}
g_{n}\left(x_{n}\right) & \longrightarrow \inf (K, g)=0, \\
d^{\prime}\left(x_{n}, K\right) & \longrightarrow 0,
\end{aligned}
$$

where $g_{n}\left(x_{n}\right)=\sup _{y \in K \cup\left\{x_{n}\right\}}\left\{\left\langle u_{n}, x_{n}-y\right\rangle+\phi\left(x_{n}\right)-\phi(y)\right\}$, $\forall n \in \mathbf{N}$. So, we deduce that 


$$
\sup _{y \in K}\left\{\left\langle u_{n}, x_{n}-y\right\rangle+\phi\left(x_{n}\right)-\phi(y)\right\} \leq \sup _{y \in K \cup\left\{x_{n}\right\}}\left\{\left\langle u_{n}, x_{n}-y\right\rangle+\phi\left(x_{n}\right)-\phi(y)\right\}=g_{n}\left(x_{n}\right)
$$

which immediately yields

$$
\limsup _{n \longrightarrow \infty} \sup _{y \in K}\left\{\left\langle u_{n}, x_{n}-y\right\rangle+\phi\left(x_{n}\right)-\phi(y)\right\} \leq 0 .
$$

Thus, there exist $0<\epsilon_{n} \longrightarrow 0$ and $u_{n} \in F\left(x_{n}\right)$ such that

$$
\sup _{y \in K}\left\{\left\langle u_{n}, x_{n}-y\right\rangle+\phi\left(x_{n}\right)-\phi(y)\right\} \leq \epsilon_{n} ;
$$

that is,

$$
\left\langle u_{n}, x_{n}-y\right\rangle+\phi\left(x_{n}\right)-\phi(y) \leq \epsilon_{n}, \quad \forall y \in K .
$$

Furthermore, from $d^{\prime}\left(x_{n}, K\right) \longrightarrow 0$ it follows that there exists $\bar{x}_{n} \in K \quad$ such that $\left\|\bar{x}_{n}-x_{n}\right\| \longrightarrow 0$. Putting $w_{n}=\bar{x}_{n}-x_{n}$, we get $x_{n}+w_{n}=\bar{x}_{n} \in K$ with $w_{n} \longrightarrow 0$. Therefore, $\left\{x_{n}\right\}$ is a LP approximating sequence for $\operatorname{GMVI}(F, \phi, K)$, and hence $x_{n} \longrightarrow x^{*}$ as $n \longrightarrow \infty$. This means that (OP) is LP well-posed.

We show that (ii) $\Rightarrow$ (i). Indeed, suppose that $(\mathrm{OP})$ is LP well-posed and $x^{*} \in K$ is the unique solution of (OP). By Lemma $2, x^{*} \in K$ is the unique solution of $\operatorname{GMVI}(F, \phi, K)$. Then, we get $\inf (K, g)=0$. Let $\left\{x_{n}\right\} \subset X$ be a LP approximating sequence for $\operatorname{GMVI}(F, \phi, K)$. Then, there exist $w_{n} \in X$ with $w_{n} \longrightarrow 0$ and $0<\epsilon_{n} \longrightarrow 0$ such that $x_{n}+w_{n} \in K$ for all $n \in \mathbf{N}$, and there exists $u_{n} \in F\left(x_{n}\right)$ such that

$$
\left\langle u_{n}, x_{n}-y\right\rangle+\phi\left(x_{n}\right)-\phi(y) \leq \epsilon_{n}, \quad \forall y \in K, n \in \mathbf{N},
$$

that is,

$$
\sup _{y \in K}\left\{\left\langle u_{n}, x_{n}-y\right\rangle+\phi\left(x_{n}\right)-\phi(y)\right\} \leq \epsilon_{n}, \quad \forall n \in \mathbf{N} .
$$

Putting $\bar{x}_{n}=x_{n}+w_{n}$ for all $n \in \mathbf{N}$, we get $\bar{x}_{n} \in K$ for all $n \in \mathbf{N}$. Then, it is easy to see that $d^{\prime}\left(x_{n}, K\right) \leq\left\|x_{n}-\bar{x}_{n}\right\|=$ $\left\|w_{n}\right\| \longrightarrow 0$. Observe that for all $n \in \mathbf{N}$,

$$
\begin{aligned}
0 & \leq g_{n}\left(x_{n}\right) \\
& =\sup _{y \in K \cup\left\{x_{n}\right\}}\left\{\left\langle u_{n}, x_{n}-y\right\rangle+\phi\left(x_{n}\right)-\phi(y)\right\} \\
& =\max \left\{\sup _{y \in K}\left\{\left\langle u_{n}, x_{n}-y\right\rangle+\phi\left(x_{n}\right)-\phi(y)\right\}, 0\right\} \\
& \leq \epsilon_{n} \longrightarrow 0 .
\end{aligned}
$$

Consequently, $\left\{x_{n}\right\}$ is a LP minimizing sequence for $(\mathrm{OP})$, and hence $x_{n} \longrightarrow x^{*}$ as $n \longrightarrow \infty$. This means that $\operatorname{GMVI}(F, \phi, K)$ is LP well-posed.

\section{Well-Posedness}

In this section, we investigate the relations between Levitin-Polyak well-posedness and Hadamard well-posedness of a generalized mixed variational inequality.

Theorem 1. Let $K$ be a nonempty, closed subset of $X$ and $F: X \longrightarrow 2^{X^{*}}$ be a nonempty set-valued mapping. Let $\phi: X \longrightarrow \mathbf{R} \cup\{+\infty\}$ be a functional. Then, $\operatorname{GMVI}(F, \phi, K)$ is $L P$ well-posed whenever $\operatorname{GMVI}(F, \phi, K)$ is Hadamard wellposed.

Proof. Suppose that $\operatorname{GMVI}(F, \phi, K)$ is Hadamard wellposed and $x^{*} \in K$ is the unique solution of $\operatorname{GMVI}(F, \phi, K)$. Let $\left\{x_{n}\right\} \subset X$ be an LP approximating sequence for $\operatorname{GMVI}(F, \phi, K)$. Then, there exist $w_{n} \in X$ with $w_{n} \longrightarrow 0$ and $0<\epsilon_{n} \longrightarrow 0$ such that $x_{n}+w_{n} \in K$ for all $n \in \mathbf{N}$, and there exists $u_{n} \in F\left(x_{n}\right)$ such that

$$
\left\langle u_{n}, x_{n}-y\right\rangle+\phi\left(x_{n}\right)-\phi(y) \leq \epsilon_{n}, \quad \forall y \in K,
$$

that is,

$$
\sup _{y \in K}\left\{\left\langle u_{n}, x_{n}-y\right\rangle+\phi\left(x_{n}\right)-\phi(y)\right\} \leq \epsilon_{n}, \quad \forall n \in N .
$$

So, it follows from $x_{n}+w_{n} \in K$ that there exists $\bar{x}_{n} \in K$ such that $x_{n}+w_{n}=\bar{x}_{n}$ for all $n \in \mathbf{N}$. Thus, we get $d^{\prime}\left(x_{n}, K\right) \leq\left\|x_{n}-\bar{x}_{n}\right\|=\left\|w_{n}\right\| \longrightarrow 0$. For each $n \in \mathbf{N}, x \in X$, we construct a sequence $\left\{\left(F_{n}, \phi_{n}, K_{n}\right)\right\}$ as follows:

$$
\begin{aligned}
\left\langle F_{n}(x), x-y\right\rangle & =\langle F(x), x-y\rangle-\epsilon_{n}, \quad \forall y \in K, \\
\phi_{n}(x) & =\phi(x)-\epsilon_{n},
\end{aligned}
$$

and $K_{n}=K \cup\left\{x_{n}\right\}$.

It is obvious that $x_{n} \in K_{n}, F_{n} \in \tau(X), \phi_{n} \in B(X)$, and $K_{n} \in C(X)$. It follows from (24)-(27) that

$$
\begin{aligned}
& \left\langle F_{n}\left(x_{n}\right), x_{n}-y\right\rangle+\phi_{n}\left(x_{n}\right)-\phi_{n}(y) \\
& =\left\langle F_{n}\left(x_{n}\right), x_{n}-y\right\rangle-\epsilon_{n}+\phi\left(x_{n}\right)-\epsilon_{n}-\left[\phi_{n}(y)-\epsilon_{n}\right] \\
& =\left\langle F_{n}\left(x_{n}\right), x_{n}-y\right\rangle+\phi\left(x_{n}\right)-\phi_{n}(y)-\epsilon_{n}, \quad \forall y \in K .
\end{aligned}
$$

Since $K_{n}=K \cup\left\{x_{n}\right\}$, it follows from (24) that

$$
\begin{aligned}
0 \leq & \mathscr{G}_{n}\left(x_{n}\right) \\
= & \sup _{y \in K_{n}} \inf \left\{\left\langle F_{n}\left(x_{n}\right), x_{n}-y\right\rangle+\phi_{n}\left(x_{n}\right)-\phi_{n}(y)\right\} \\
= & \sup _{y \in K_{n}} \inf \left\{\left\langle F_{n}\left(x_{n}\right), x_{n}-y\right\rangle+\phi\left(x_{n}\right)-\phi_{n}(y)-\epsilon_{n}\right\} \\
= & \sup _{y \in K_{n}} \inf _{u \in F\left(x_{n}\right)}\left\{\left\langle u, x_{n}-y\right\rangle+\phi\left(x_{n}\right)-\phi_{n}(y)-\epsilon_{n}\right\} \\
\leq & \sup _{y \in K_{n}}\left\{\left\langle u, x_{n}-y\right\rangle+\phi\left(x_{n}\right)-\phi_{n}(y)-\epsilon_{n}\right\} \leq 0 .
\end{aligned}
$$

That is, $\mathscr{G}_{n}\left(x_{n}\right)=0$ for all $n \in \mathbf{N}$. So, it follows from Lemma 2 that $x_{n} \in S\left(F_{n}, \phi_{n}, K_{n}\right)$ for all $n \in \mathbf{N}$. From (3) and (26), we have $d\left(F_{n}, F\right)=\left|\epsilon_{n}\right| \longrightarrow 0$. Again from (6) and (27), we have $d_{1}\left(\phi_{n}, \phi\right) \longrightarrow 0$. We also obtain that $\mathscr{H}\left(K_{n}, K\right)$ $=d^{\prime}\left(x_{n}, K\right) \longrightarrow 0$. Thus, we have $\rho\left(\left(F_{n}, \phi_{n}, K_{n}\right),(F, \phi, K)\right)$ $\longrightarrow 0$. Since $\operatorname{GMVI}(F, \phi, K)$ is Hadamard well-posed, we know that $\left\{x_{n}\right\}$ converges strongly to the unique solution $x^{*}$ 
of $\operatorname{GMVI}(F, \phi, K)$. Thus, $\operatorname{GMVI}(F, \phi, K)$ is LP well-posed. The proof is complete.

Next, we have the following result which can be regarded as the reverse of Theorem 1 under the uniform continuity of the function $\phi$.

Theorem 2. Let $K$ be a nonempty, closed subset of $X$ and $F: X \longrightarrow 2^{X^{*}}$ be a nonempty set-valued mapping. Let $\phi: X \longrightarrow \mathbf{R} \cup\{+\infty\}$ be uniformly continuous on $X$. Then, $\operatorname{GMVI}(F, \phi, K)$ is Hadamard well-posed whenever $\operatorname{GMVI}(F, \phi, K)$ is LP well-posed.

Proof. Suppose that $\operatorname{GMVI}(F, \phi, K)$ is LP well-posed and $x^{*} \in K$ is the unique solution of $\operatorname{GMVI}(F, \phi, K)$. Let

$$
g(x)=\sup _{y \in K} \inf \{\langle F(x), x-y\rangle+\phi(x)-\phi(y)\}, \quad \forall x \in X .
$$

Since $\operatorname{GMVI}(F, \phi, K)$ has the unique solution $x^{*} \in K$, by Lemma 2, we know that (OP) has the unique solution $x^{*} \in K$. That is, $\inf (K, g)=0$ and $\arg \min (K, g)=\left\{x^{*}\right\}$. Let $\left\{\left(F_{n}, \phi_{n}, K_{n}\right)\right\} \in \tau(X) \times B(X) \times C(X),\left(F_{n}, \phi_{n}, K_{n}\right) \quad$ converges to $(F, \phi, K)$ and $x_{n} \in S\left(F_{n}, \phi_{n}, K_{n}\right)$, where $K \cup\left\{x_{n}\right\} \subseteq K_{n}$. So, it follows from $x_{n} \in S\left(F_{n}, \phi_{n}, K_{n}\right)$ that there exists $\widetilde{u}_{n} \in F_{n}\left(x_{n}\right)$ such that

$$
\left\langle\widetilde{u}_{n}, x_{n}-y\right\rangle+\phi_{n}\left(x_{n}\right)-\phi_{n}(y) \leq 0, \quad \forall y \in K_{n},
$$

which immediately yields

$$
\left\langle\tilde{u}_{n}, x_{n}-y\right\rangle+\phi_{n}\left(x_{n}\right)-\phi_{n}(y) \leq 0, \quad \forall y \in K .
$$

For any $x \in X$, let

$\mathscr{G}_{n}(x)=\sup _{y \in K_{n}} \inf \left\{\left\langle F_{n}(x), x-y\right\rangle+\phi_{n}(x)-\phi_{n}(y)\right\}, \quad \forall x \in X$.

From Lemma 1, it is easy to see that

(i) $\mathscr{G}_{n}(x) \geq 0, \forall x \in K_{n}$;

(ii) for any $x \in K_{n}, \mathscr{G}_{n}(x)=0 \Longleftrightarrow x \in S\left(F_{n}, \phi_{n}, K_{n}\right)$.

It follows from (ii) and $x_{n} \in S\left(F_{n}, \phi_{n}, K_{n}\right)$ that $\mathscr{G}_{n}\left(x_{n}\right)=\inf \left(K_{n}, \mathscr{G}\right)=0$. On the other hand, note that $\rho\left(\left(F_{n}, \phi_{n}, K_{n}\right),(F, \phi, K)\right) \longrightarrow 0$. Then, we deduce that $d\left(F_{n}, F\right) \longrightarrow 0, d_{1}\left(\phi_{n}, \phi\right) \longrightarrow 0$ and $\mathscr{H}\left(K_{n}, K\right) \longrightarrow 0$. Since $d\left(F_{n}, F\right) \longrightarrow 0$, it follows from (3) that there exists $0<\epsilon_{n} \longrightarrow 0$ such that for any $x \in X$,

$$
\left\langle F_{n}(x), x-y\right\rangle=\langle F(x), x-y\rangle-\epsilon_{n}, \quad \forall y \in K .
$$

In particular, we have

$$
\left\langle F_{n}\left(x_{n}\right), x_{n}-y\right\rangle=\left\langle F\left(x_{n}\right), x_{n}-y\right\rangle-\epsilon_{n}, \quad \forall y \in K \text {. }
$$

From $\tilde{u}_{n} \in F_{n}\left(x_{n}\right)$ it follows that there exists $u_{n} \in F\left(x_{n}\right)$ such that

$$
\left\langle\widetilde{u}_{n}, x_{n}-y\right\rangle=\left\langle u_{n}, x_{n}-y\right\rangle-\epsilon_{n}, \quad \forall y \in K .
$$

This together with (32) leads to

$$
\left\langle u_{n}, x_{n}-y\right\rangle+\phi_{n}\left(x_{n}\right)-\phi_{n}(y) \leq \epsilon_{n}, \quad \forall y \in K,
$$

which can be rewritten as follows:

$$
\left\langle u_{n}, x_{n}-y\right\rangle+\phi\left(x_{n}\right)-\phi(y) \leq \epsilon_{n}+\phi\left(x_{n}\right)-\phi_{n}\left(x_{n}\right)-\left(\phi(y)-\phi_{n}(y)\right), \quad \forall y \in K
$$

Next, we claim that

$$
\lim _{n \longrightarrow \infty}\left|\phi\left(x_{n}\right)-\phi_{n}\left(x_{n}\right)-\left(\phi(y)-\phi_{n}(y)\right)\right|=0, \quad \text { uniformly for } y \in X
$$

As a matter of fact, for any $\delta>0$, since $d_{1}\left(\phi_{n}, \phi\right)=\sup _{x \in X}\left|\phi_{n}(x)-\phi(x)\right| \longrightarrow 0$, there exists an integer $N \geq 1$ such that for all $n \geq N$,

$$
\sup _{x \in X}\left|\phi_{n}(x)-\phi(x)\right| \leq \delta \text {. }
$$

It follows that for any $x \in X$,

$$
\phi(x)-\delta \leq \phi_{n}(x) \leq \phi(x)+\delta, \quad n \geq N .
$$

So, for any $x, y \in X$, we have

$$
\begin{aligned}
\phi_{n}(x)-\phi_{n}(y) & \leq \phi(x)+\delta-(\phi(y)-\delta) \\
& =\phi(x)-\phi(y)+2 \delta, \quad n \geq N .
\end{aligned}
$$

Meantime, we also have

$$
\begin{aligned}
\phi(x)-\phi(y)-2 \delta & =\phi(x)-\delta-(\phi(y)+\delta) \\
& \leq \phi_{n}(x)-\phi_{n}(y), \quad n \geq N .
\end{aligned}
$$

Then, for any $x, y \in X$,

$\left|\phi_{n}(x)-\phi_{n}(y)-(\phi(x)-\phi(y))\right| \leq 2 \delta, \quad n \geq N$.

In particular, for any $y \in X$, we get

$\left|\phi_{n}\left(x_{n}\right)-\phi_{n}(y)-\left(\phi\left(x_{n}\right)-\phi(y)\right)\right| \leq 2 \delta, \quad n \geq N$.

This means that (39) holds.

Finally, from (38) and (39) and $0<\epsilon_{n} \longrightarrow 0$, we have 


$$
\begin{aligned}
0 & \leq g_{n}\left(x_{n}\right)=\sup _{y \in K \cup\left\{x_{n}\right\}}\left\{\left\langle u_{n}, x_{n}-y\right\rangle+\phi\left(x_{n}\right)-\phi(y)\right\} \\
& \leq \max \left\{\sup _{y \in K}\left\{\epsilon_{n}+\phi\left(x_{n}\right)-\phi_{n}\left(x_{n}\right)-\left(\phi(y)-\phi_{n}(y)\right)\right\}, 0\right\} \\
& \leq \epsilon_{n}+\sup _{y \in K}\left|\phi\left(x_{n}\right)-\phi_{n}\left(x_{n}\right)-\left(\phi(y)-\phi_{n}(y)\right)\right| \longrightarrow 0 .
\end{aligned}
$$

Since $K_{n} \longrightarrow K$ in the Hausdorff metric and $x_{n} \in K_{n}$, we have $d^{\prime}\left(x_{n}, K\right) \longrightarrow 0$. Thus, $\left\{x_{n}\right\}$ is an LP minimizing sequence for (OP). Since $\operatorname{GMVI}(F, \phi, K)$ is LP well-posed, according to Lemma 3, we know that (OP) is LP well-posed. Therefore, $x_{n} \longrightarrow x^{*}$ as $n \longrightarrow \infty$. So, it follows that $\operatorname{GMVI}(F, \phi, K)$ is Hadamard well-posed. The proof is complete.

Remark 1. Theorems 1 and 2 improve, extend, and develop Theorems 3.1 and 3.2 in [12] to a great extent because the generalized mixed variational inequality considered in Theorems 1 and 2 is more general than the general mixed variational inequality considered in [[12], Theorems 3.1 and 3.2].

\section{Metric Characterization and Conditions for Hadamard Well-Posedness}

In this section, we derive the metric characterization of Hadamard well-posedness for a generalized mixed variational inequality and prove that under suitable conditions, the Hadamard well-posedness of a generalized mixed variational inequality is equivalent to the existence and uniqueness of its solutions.

To characterize the Hadamard well-posedness for a generalized mixed variational inequality $\operatorname{GMVI}(F, \phi, K)$, we define

$$
\Omega(\epsilon)=\left\{x \in X: d^{\prime}(x, K) \leq \epsilon \text {, and there exists } u \in F(x) \text { such that } \forall y \in K,\langle u, x-y\rangle+\phi(x)-\phi(y) \leq \epsilon\right\}, \quad \forall \epsilon \geq 0
$$

Theorem 3. Let $(F, \phi, K) \in \tau(X) \times B(X) \times C(X), K$ be convex, $F: X \longrightarrow 2^{X^{*}}$ be a nonempty compact-valued mapping which is $\mathscr{H}$-hemicontinuous and monotone, and $\phi: X \longrightarrow \mathbf{R} \cup\{+\infty\}$ be proper, convex, and uniformly continuous on $X$. Then, GMVI $(F, \phi, K)$ is Hadamard well-posed if and only if

$$
\Omega(\epsilon) \neq \varnothing, \quad \forall \epsilon>0 \text { and } \operatorname{diam}(\Omega(\epsilon)) \longrightarrow 0 \text { as } \epsilon \longrightarrow 0 .
$$

Proof. Assume that $\operatorname{GMVI}(F, \phi, K)$ is Hadamard wellposed. Then, $\operatorname{GMVI}(F, \phi, K)$ has a unique solution which lies in $\Omega(\epsilon)$ for all $\epsilon>0$. Put $x_{0} \in S(F, \phi, K)$. Obviously, $x_{0} \in \Omega(\epsilon)$ for all $\epsilon>0$. If $\operatorname{diam}(\Omega(\epsilon)) \nrightarrow 0$ as $\epsilon \longrightarrow 0$, then for some $\delta>0,0<\epsilon_{n} \longrightarrow 0$ such that for $n$ sufficiently large,

$$
\operatorname{diam}\left(\Omega\left(\epsilon_{n}\right)\right)>\delta>0 .
$$

Thus, we can find points $x_{n} \in \Omega\left(\epsilon_{n}\right)$ such that

$$
\left\|x_{n}-x_{0}\right\|>\frac{\delta}{2}
$$

Since $x_{n} \in \Omega\left(\epsilon_{n}\right)$, we have

$$
d^{\prime}\left(x_{n}, K\right) \leq \epsilon_{n}
$$

and there exists $u_{n} \in F\left(x_{n}\right)$ such that

$$
\left\langle u_{n}, x_{n}-y\right\rangle+\phi\left(x_{n}\right)-\phi(y) \leq \epsilon_{n}, \quad \forall y \in K .
$$

Now, we construct a sequence $\left\{\left(F_{n}, \phi_{n}, K_{n}\right)\right\}$ as follows:

$$
\begin{aligned}
\left\langle F_{n}(x), x-y\right\rangle & =\left\langle F_{n}(x), x-y\right\rangle-\epsilon_{n}, \quad \forall y \in K, \\
\phi_{n}(x) & =\phi(x)-\epsilon_{n}, \\
K_{n} & =K \bigcup\left\{x_{n}\right\} .
\end{aligned}
$$

It is obvious that $x_{n} \in K_{n}, F_{n} \tau(X), \phi_{n} \in B(X)$, and $K_{n} \in C(X)$. By the similar argument to that in the proof of Theorem 1, we have $x_{n} \in S\left(F_{n}, \phi_{n}, K_{n}\right)$. Observe that $d\left(F_{n}, F\right)=\left|\epsilon_{n}\right| \longrightarrow 0, d_{1}\left(\phi_{n}, \phi\right) \longrightarrow 0$, and $\mathscr{H}\left(K_{n}, K\right)=$ $d^{\prime}\left(x_{n}, K\right) \longrightarrow 0$. Thus, we have $\rho\left(\left(F_{n}, \phi_{n}, K_{n}\right)\right.$, $(F, \phi, K)) \longrightarrow 0$. Since $\operatorname{GMVI}(F, \phi, K)$ is Hadamard wellposed, one has $x_{n} \longrightarrow x_{0}$, a contradiction to (50).

Conversely, suppose that condition (48) holds. Let $\left(F_{n}, \phi_{n}, K_{n}\right) \longrightarrow(F, \phi, K)$, and $x_{n} \in S\left(F_{n}, \phi_{n}, K_{n}\right)$, where $K \cup\left\{x_{n}\right\} \subseteq K_{n}, n=1,2, \ldots$ So, it follows from $x_{n} \in S\left(F_{n}, \phi_{n}, K_{n}\right)$ that there exists $\widetilde{u}_{n} \in F_{n}\left(x_{n}\right)$ such that

$$
\left\langle\widetilde{u}_{n}, x_{n}-y\right\rangle+\phi_{n}\left(x_{n}\right)-\phi_{n}(y) \leq 0, \quad \forall y \in K_{n},
$$

which immediately yields

$$
\left\langle\tilde{u}_{n}, x_{n}-y\right\rangle+\phi_{n}\left(x_{n}\right)-\phi_{n}(y) \leq 0, \quad \forall y \in K .
$$

Furthermore, note that $\rho\left(\left(F_{n}, \phi_{n}, K_{n}\right),(F, \phi, K)\right) \longrightarrow 0$. Then, we deduce that $d\left(F_{n}, F\right) \longrightarrow 0, d_{1}\left(\phi_{n}, \phi\right) \longrightarrow 0$ and $\mathscr{H}\left(K_{n}, K\right) \longrightarrow 0$. Since $d\left(F_{n}, F\right) \longrightarrow 0$, it follows from (3) that there exists $0<\epsilon_{n}^{\prime} \longrightarrow 0$ such that for any $x \in X$,

$$
\left\langle F_{n}(x), x-y\right\rangle=\langle F(x), x-y\rangle-\epsilon_{n}^{\prime}, \quad \forall y \in K .
$$

In particular, we have

$$
\left\langle F_{n}\left(x_{n}\right), x_{n}-y\right\rangle=\left\langle F\left(x_{n}\right), x_{n}-y\right\rangle-\epsilon_{n}^{\prime}, \quad \forall y \in K .
$$


From $\widetilde{u}_{n} \in F_{n}\left(x_{n}\right)$, it follows that there exists $u_{n} \in F\left(x_{n}\right)$ such that

$$
\left\langle\tilde{u}_{n}, x_{n}-y\right\rangle=\left\langle u_{n}, x_{n}-y\right\rangle-\epsilon_{n}^{\prime}, \quad \forall y \in K .
$$

This together with (55) leads to

$$
\left\langle u_{n}, x_{n}-y\right\rangle+\phi_{n}\left(x_{n}\right)-\phi_{n}(y) \leq \epsilon_{n}^{\prime}, \quad \forall y \in K,
$$

which can be rewritten as follows:

$$
\left\langle u_{n}, x_{n}-y\right\rangle+\phi\left(x_{n}\right)-\phi(y) \leq \epsilon_{n}^{\prime}+\phi\left(x_{n}\right)-\phi_{n}\left(x_{n}\right)-\left(\phi(y)-\phi_{n}(y)\right), \quad \forall y \in K
$$

Repeating the same argument as that of (39) in the proof of Theorem 2, we get

$$
\lim _{n \longrightarrow \infty}\left|\phi\left(x_{n}\right)-\phi_{n}\left(x_{n}\right)-\left(\phi(y)-\phi_{n}(y)\right)\right|=0, \quad \text { uniformly for } y \in X \text {. }
$$

Taking into account that $K_{n} \longrightarrow K$ in the Hausdorff metric and $x_{n} \in K_{n}$, we have $d^{\prime}\left(x_{n}, K\right) \longrightarrow 0$. Thus, there exists $0<\epsilon_{n}^{*} \longrightarrow 0$ such that $d^{\prime}\left(x_{n}, K\right) \leq \epsilon_{n}^{*}$ and

$$
\sup _{y \in X}\left|\phi\left(x_{n}\right)-\phi_{n}\left(x_{n}\right)-\left(\phi(y)-\phi_{n}(y)\right)\right| \leq \epsilon_{n}^{*}, \quad \forall n \in N .
$$

Set $\epsilon_{n}=\epsilon_{n}^{\prime}+\epsilon_{n}^{*}$. Then, it follows from (60) that

$$
\left\langle u_{n}, x_{n}-y\right\rangle+\phi\left(x_{n}\right)-\phi(y) \leq \epsilon_{n}, \quad \forall y \in K .
$$

This means that $x_{n} \in \Omega\left(\epsilon_{n}\right)$ for all $n \in \mathbf{N}$. From (48), we know that $\left\{x_{n}\right\}$ is a Cauchy sequence and so it converges strongly a point $\bar{x} \in K$. Since $F$ is monotone and $\phi$ is lower semicontinuous, it follows from (63) that for any $y \in K, v \in F(y)$,

$$
\begin{aligned}
\langle v, \bar{x}-y\rangle+\phi(\bar{x})-\phi(y) \\
\quad \cdot \liminf _{n \longrightarrow \infty}\left\{\left\langle v, x_{n}-y\right\rangle+\phi\left(x_{n}\right)-\phi(y)\right\} \\
\leq \liminf _{n \longrightarrow \infty}\left\{\left\langle u_{n}, x_{n}-y\right\rangle+\phi\left(x_{n}\right)-\phi(y)\right\} \\
\leq \liminf _{n \longrightarrow \infty} \epsilon_{n}=0 .
\end{aligned}
$$

So, from Proposition 1 , it is easy to see that $\bar{x}$ solves $\operatorname{GMVI}(F, \phi, K)$.

To complete the proof, we need only to prove that $\operatorname{GMVI}(F, \phi, K)$ has a unique solution. Assume by contradiction that $\operatorname{GMVI}(F, \phi, K)$ has two distinct solutions $x_{1}$ and $x_{2}$ in $K$. Then, it is easy to see that $x_{1}, x_{2} \in \Omega(\epsilon)$ for all $\epsilon>0$ and

$$
0<\left\|x_{1}-x_{2}\right\| \leq \operatorname{diam}(\Omega(\epsilon)) \longrightarrow 0,
$$

a contradiction to (48). The proof is complete.

Next, we prove that the Hadamard well-posedness of a generalized mixed variational inequality is equivalent to the existence and uniqueness of its solutions under suitable conditions.
Theorem 4. Let $(F, \phi, K) \in \tau(X) \times B(X) \times C(X), K$ be convex, $F: X \longrightarrow 2^{X^{*}}$ be a nonempty compact-valued mapping which is $\mathscr{H}$-hemicontinuous and monotone, and $\phi: X \longrightarrow \mathbf{R} \cup\{+\infty\}$ be proper, convex, and uniformly continuous on X. Then, GMVI $(F, \phi, K)$ is Hadamard well-posed if and only if it has a unique solution.

Proof. The necessity is obvious. For the sufficiency, suppose that $\operatorname{GMVI}(F, \phi, K)$ has a unique solution $x^{*}$. If $\operatorname{GMVI}(F, \phi, K)$ is not Hadamard well-posed, then there exists $\left\{\left(F_{n}, \phi_{n}, K_{n}\right)\right\} \subset M$ converging to $(F, \phi, K)$ with $x_{n} \in S\left(F_{n}, \phi_{n}, K_{n}\right)$ such that $\left\{x_{n}\right\}$ do not converge to $x^{*}$, where $K \cup\left\{x_{n}\right\} \subseteq K_{n}, n=1,2, \ldots$. So, it follows from $x_{n} \in S\left(F_{n}, \phi_{n}, K_{n}\right)$ that there exists $\widetilde{u}_{n} \in F_{n}\left(x_{n}\right)$ such that

$$
\left\langle\widetilde{u}_{n}, x_{n}-y\right\rangle+\phi_{n}\left(x_{n}\right)-\phi_{n}(y) \leq 0, \quad \forall y \in K_{n},
$$

which immediately yields

$$
\left\langle\tilde{u}_{n}, x_{n}-y\right\rangle+\phi_{n}\left(x_{n}\right)-\phi_{n}(y) \leq 0, \quad \forall y \in K .
$$

Furthermore, note that $\rho\left(\left(F_{n}, \phi_{n}, K_{n}\right),(F, \phi, K)\right) \longrightarrow 0$. Then, we deduce that $d\left(F_{n}, F\right) \longrightarrow 0, d_{1}\left(\phi_{n}, \phi\right) \longrightarrow 0$ and $\mathscr{H}\left(K_{n}, K\right) \longrightarrow 0$. Since $d\left(F_{n}, F\right) \longrightarrow 0$, it follows from (3) that there exists $0<\epsilon_{n}^{\prime} \longrightarrow 0$ such that for any $x \in X$,

$$
\left\langle F_{n}(x), x-y\right\rangle=\langle F(x), x-y\rangle-\epsilon_{n}^{\prime}, \quad \forall y \in K .
$$

In particular, we have

$$
\left\langle F_{n}\left(x_{n}\right), x_{n}-y\right\rangle=\left\langle F\left(x_{n}\right), x_{n}-y\right\rangle-\epsilon_{n}^{\prime}, \quad \forall y \in K .
$$

From $\tilde{u}_{n} \in F_{n}\left(x_{n}\right)$, it follows that there exists $u_{n} \in F\left(x_{n}\right)$ such that

$$
\left\langle\tilde{u}_{n}, x_{n}-y\right\rangle=\left\langle u_{n}, x_{n}-y\right\rangle-\epsilon_{n}^{\prime}, \quad \forall y \in K .
$$

This together with (67) leads to

$$
\left\langle u_{n}, x_{n}-y\right\rangle+\phi\left(x_{n}\right)-\phi(y) \leq \epsilon_{n}^{\prime}+\phi\left(x_{n}\right)-\phi_{n}\left(x_{n}\right)-\left(\phi(y)-\phi_{n}(y)\right), \quad \forall y \in K .
$$


Repeating the same argument as that of (63) in the proof of Theorem 3, we obtain that there exists $0<\epsilon_{n} \longrightarrow 0$ such that $d^{\prime}\left(x_{n}, K\right) \leq \epsilon_{n}$ and

$$
\left\langle u_{n}, x_{n}-y\right\rangle+\phi\left(x_{n}\right)-\phi(y) \leq \epsilon_{n}, \quad \forall y \in K .
$$

From $d^{\prime}\left(x_{n}, K\right) \leq \epsilon_{n}<\epsilon_{n}+(1 / n)$, it follows that there exists $\bar{x}_{n} \in K$ such that $\left\|\bar{x}_{n}-x_{n}\right\|<\epsilon_{n}+(1 / n) \longrightarrow 0$. Putting $w_{n}=\bar{x}_{n}-x_{n}$, we get $\bar{x}_{n}=w_{n}+x_{n}$ with $w_{n} \longrightarrow 0$.

We claim that $\left\{x_{n}\right\}$ is bounded. As a matter of fact, if $\left\{x_{n}\right\}$ is unbounded, then $\left\{\bar{x}_{n}\right\}$ is an unbounded sequence in $K$. Without loss of generality, we may assume that $\left\|\bar{x}_{n}\right\| \longrightarrow+\infty$. Let

$$
t_{n}=\frac{1}{\left\|\bar{x}_{n}-x^{*}\right\|}, \quad z_{n}=x^{*}+t_{n}\left(\bar{x}_{n}-x^{*}\right) .
$$

Without loss of generality, we may assume that $t_{n} \in(0,1]$ and $z_{n} \rightarrow z\left(\neq x^{*}\right)$. Then, we have for each $y \in K, v \in F(y)$,

$$
\begin{aligned}
\langle v, z-y\rangle= & \left\langle v, z-z_{n}\right\rangle+\left\langle v, z_{n}-x^{*}\right\rangle+\left\langle v, x^{*}-y\right\rangle \\
= & \left\langle v, z-z_{n}\right\rangle+t_{n}\left\langle v, \bar{x}_{n}-x^{*}\right\rangle+\left\langle v, x^{*}-y\right\rangle \\
= & \left\langle v, z-z_{n}\right\rangle+t_{n}\left\langle v, x_{n}+w_{n}-x^{*}\right\rangle+\left\langle v, x^{*}-y\right\rangle \\
= & \left\langle v, z-z_{n}\right\rangle+t_{n}\left\langle v, x_{n}-y\right\rangle+\left(1-t_{n}\right)\left\langle v, x^{*}-y\right\rangle \\
& +t_{n}\left\langle v, w_{n}\right\rangle .
\end{aligned}
$$

Since $x^{*}$ is the unique solution of $\operatorname{GMVI}(F, \phi, K)$, there exists $u^{*} \in F\left(x^{*}\right)$ such that

$$
\left\langle u^{*}, x^{*}-y\right\rangle+\phi\left(x^{*}\right)-\phi(y) \leq 0, \quad \forall y \in K .
$$

Since $F$ is monotone, we have

$$
\begin{gathered}
\left\langle v, x^{*}-y\right\rangle \leq\left\langle u^{*}, x^{*}-y\right\rangle, \\
\left\langle v, x_{n}-y\right\rangle \leq\left\langle u_{n}, x_{n}-y\right\rangle .
\end{gathered}
$$

It follows from (72)-(76) and the convexity of $\phi$ that for all $v \in F(y)$,

$$
\begin{aligned}
& \langle v, z-y\rangle \\
& \leq\left\langle v, z-z_{n}\right\rangle+t_{n} \phi(y)-t_{n} \phi\left(x_{n}\right)+t_{n} \epsilon_{n}+\left(1-t_{n}\right)\left(\phi(y)-\phi\left(x^{*}\right)\right)+t_{n}\left\langle v, w_{n}\right\rangle \\
& =\left\langle v, z-z_{n}\right\rangle+\phi(y)-\left[t_{n} \phi\left(x_{n}\right)+\left(1-t_{n}\right) \phi\left(x^{*}\right)\right]+t_{n} \epsilon_{n}+t_{n}\left\langle v, w_{n}\right\rangle \\
& =\left\langle v, z-z_{n}\right\rangle+\phi(y)-\left[t_{n} \phi\left(\bar{x}_{n}\right)+\left(1-t_{n}\right) \phi\left(x^{*}\right)+t_{n} \phi\left(x_{n}\right)-t_{n} \phi\left(\bar{x}_{n}\right)\right]+t_{n} \epsilon_{n}+t_{n}\left\langle v, w_{n}\right\rangle \\
& \leq\left\langle v, z-z_{n}\right\rangle+\phi(y)-\phi\left(z_{n}\right)-t_{n}\left[\phi\left(x_{n}\right)-\phi\left(\bar{x}_{n}\right)\right]+t_{n} \epsilon_{n}+t_{n}\left\langle v, w_{n}\right\rangle, \quad \forall y \in K .
\end{aligned}
$$

Since $\phi$ is uniformly continuous, we have

$$
\begin{aligned}
& \langle v, z-y\rangle \\
& \leq \liminf _{n \longrightarrow \infty}\left\{\langle v, z-y\rangle+\phi(y)-\phi\left(z_{n}\right)-t_{n}\left[\phi\left(x_{n}\right)-\phi\left(\bar{x}_{n}\right)\right]\right. \\
& \left.\quad+t_{n} \epsilon_{n}+t_{n}\left\langle v, w_{n}\right\rangle\right\} \\
& \leq \phi(y)-\phi(z), \quad \forall y \in K .
\end{aligned}
$$

This together with Proposition 1 implies that $z$ solves $\operatorname{GMVI}(F, \phi, K)$, a contradiction. Thus, $\left\{x_{n}\right\}$ is bounded.

Next, we claim that $x_{n} \longrightarrow x^{*}$ as $n \longrightarrow \infty$. Let $\left\{x_{n_{k}}\right\}$ be any subsequence of $\left\{x_{n}\right\}$ such that $x_{n_{k}} \longrightarrow \bar{x}$ as $k \longrightarrow \infty$. Clearly, $\bar{x} \in K$. It follows from (72) that

$$
\left\langle u_{n_{k}}, x_{n_{k}}-y\right\rangle+\phi\left(x_{n_{k}}\right)-\phi(y) \leq \epsilon_{n_{k}}, \quad \forall y \in K .
$$

Since $F$ is monotone and $\phi$ is uniformly continuous, we have

$$
\begin{aligned}
& \langle v, \bar{x}-y\rangle+\phi(\bar{x})-\phi(y) \\
& =\liminf _{k \longrightarrow \infty}\left\{\left\langle v, x_{n_{k}}-y\right\rangle+\phi\left(x_{n_{k}}\right)-\phi(y)\right\} \\
& \leq \liminf _{k \longrightarrow \infty}\left\{\left\langle u_{n_{k}}, x_{n_{k}}-y\right\rangle+\phi\left(x_{n_{k}}\right)-\phi(y)\right\} \\
& \leq \liminf _{k \rightarrow \infty} \epsilon_{n_{k}}=0, \quad \forall y \in K, v \in F(y) .
\end{aligned}
$$

This together with Proposition 1 implies that $\bar{x}$ solves $\operatorname{GMVI}(F, \phi, K)$. Since $\operatorname{GMVI}(F, \phi, K)$ has a unique solution $x^{*}$, we have $\bar{x}=x^{*}$. Thus, $x_{n} \longrightarrow x^{*}$, which reaches a contradiction. So, $\operatorname{GMVI}(F, \phi, K)$ is Hadamard well-posed. The proof is complete.

\section{Concluding Remarks}

Theorems 3 and 4 improve, extend, and develop Theorems 4.1 and 4.2 in [12] to a great extent because the generalized mixed variational inequality considered in Theorems 3 and 4 is more general than the general mixed variational inequality considered in ([12], Theorems 4.1 and 4.2). In addition, Theorems 3 and 4 also improve, extend, and develop Theorems 3.1 and $6.1 \mathrm{in}$ [7] and Theorems 3.1 and 6.1 in [20] to a great extent because Levitin-Polyak well-posedness of a generalized mixed variational inequality is replaced by Hadamard well-posedness of a generalized mixed variational inequality.

\section{Data Availability}

All data generated or analyzed during this study are included in this published article.

\section{Conflicts of Interest}

The authors declare that they have no conflicts of interest. 


\section{Authors' Contributions}

All authors contributed equally to the writing of this paper. All authors read and approved the final manuscript.

\section{Acknowledgments}

The first author was partially supported by the 2020 Shanghai Leading Talents Program of the Shanghai Municipal Human Resources and Social Security Bureau (20LJ2006100), the Innovation Program of Shanghai Municipal Education Commission (15ZZ068), and the Program for Outstanding Academic Leaders in Shanghai City (15XD1503100). The second author was supported in part by the grand from NKUST and KMU joint R\&D Project (110KK004) and MOST Project in Taiwan (110-2410-H-037 -001). The third author was partially supported by the grant MOST 104-2115-M-037-001 and the grant from Research Center for Nonlinear Analysis and Optimization, Kaohsiung Medical University.

\section{References}

[1] A. N. Tykhonov, "On the stability of the functional optimization problem," USSR Journal of Computational Mathematics and Mathematical Physics, vol. 6, pp. 631-634, 1966.

[2] R. Lucchetti and F. Patrone, "Hadamard and Tyhonov wellposedness of a certain class of convex functions," Journal of Mathematical Analysis and Applications, vol. 88, no. 1, pp. 204-215, 1982.

[3] R. Lucchetti and F. Patrone, "A characterization of tyhonov well-posedness for minimum problems, with applications to variational inequalities(*)," Numerical Functional Analysis and Optimization, vol. 3, no. 4, pp. 461-476, 1981.

[4] M. B. Lignola and J. Morgan, "Approximate solutions and $\alpha$-well-posedness for variational inequalities and nash equilibria," in Decision and Control in Management Science, G. Zaccour, Ed., Kluwer, pp. 367-377, Dordrecht, Netherlands, 2002.

[5] I. Del Prete, M. B. Lignola, and J. Morgan, "New concepts of well-posedness for optimization problems with variational inequality constraints," Journal of Inequalities in Pure and Applied Mathematics, vol. 4, no. 1, p. 5, 2003.

[6] Y.-P. Fang, N.-J. Huang, and J.-C. Yao, "Well-posedness of mixed variational inequalities, inclusion problems and fixed point problems," Journal of Global Optimization, vol. 41, no. 1, pp. 117-133, 2008.

[7] L. C. Ceng and J. C. Yao, "Well-posedness of generalized mixed variational inequalities, inclusion problems and fixedpoint problems," Nonlinear Analysis: Theory, Methods \& Applications, vol. 69, no. 12, pp. 4585-4603, 2008.

[8] L. C. Ceng, N. Hadjisavvas, S. Schaible, and J. C. Yao, "Wellposedness for mixed quasivariational-like inequalities," Journal of Optimization Theory and Applications, vol. 139, no. 1, pp. 109-125, 2008.

[9] L.-C. Ceng, H. Gupta, and C.-F. Wen, "Well-posedness by perturbations of variational-hemivariational inequalities with perturbations," Filomat, vol. 26, no. 5, pp. 881-895, 2012.

[10] L. C. Ceng, N. C. Wang, and J. C. Yao, "Well-posedness for a class of strongly mixed variational-hemivariational inequalities with perturbations," Journal of Applied Mathematics, vol. 2012, Article ID 712306, 21 pages, 2012.

[11] Y.-P. Fang, N.-J. Huang, and J.-C. Yao, "Well-posedness by perturbations of mixed variational inequalities in Banach spaces," European Journal of Operational Research, vol. 201, no. 3, pp. 682-692, 2010.

[12] X.-b. Li and F.-q. Xia, "Hadamard well-posedness of a general mixed variational inequality in Banach space," Journal of Global Optimization, vol. 56, no. 4, pp. 1617-1629, 2013.

[13] L.-C. Ceng, Y.-Y. Lur, and C.-F. Wen, "Well-posedness for generalized variational-hemivariational inequalities with perturbations in reflexive Banach spaces," Tamkang Journal of Mathematics, vol. 48, no. 4, pp. 345-364, 2017.

[14] L.-C. Ceng and C.-F. Wen, "Levitin-Polyak well-posedness of completely generalized mixed variational inequalities in reflexive Banach spaces," Tamkang Journal of Mathematics, vol. 48, no. 1, pp. 95-121, 2017.

[15] L. C. Ceng, F. Q. Xia, and J. C. Yao, "Levitin-Polyak wellposedness of noncompact generalized mixed variational inequalities in reflexive Banach spaces," Pure and Applied Functional Analysis, vol. 1, pp. 475-503, 2016.

[16] F. Q. Xia and C. F. Wen, "Levitin-Polyak well-posedness of generalized variational inequality with generalized mixed variational inequality constraint," Journal of Nonlinear Convex Analysis, vol. 16, pp. 2087-2101, 2015.

[17] L.-C. Ceng, Y.-C. Liou, J.-C. Yao, Y. Yao, and C.-H. Lo, "Wellposedness for systems of generalized mixed quasivariational inclusion problems and optimization problems with constraints," The Journal of Nonlinear Science and Applications, vol. 10, no. 10, pp. 5373-5392, 2017.

[18] L.-C. Ceng, Y.-C. Liou, J.-C. Yao, and Y. Yao, "Well-posedness for systems of time-dependent hemivariational inequalities in Banach spaces," The Journal of Nonlinear Science and Applications, vol. 10, no. 8, pp. 4318-4336, 2017.

[19] L.-C. Ceng, Y.-C. Liou, and C.-F. Wen, "On the well-posedness of generalized hemivariational inequalities and inclusion problems in Banach spaces," The Journal of Nonlinear Science and Applications, vol. 9, no. 6, pp. 3879-3891, 2016.

[20] X.-b. Li and F.-q. Xia, "Levitin-Polyak well-posedness of a generalized mixed variational inequality in Banach spaces," Nonlinear Analysis: Theory, Methods \& Applications, vol. 75, no. 4, pp. 2139-2153, 2012.

[21] H. Yang and J. Yu, "Unified approaches to well-posedness with some applications," Journal of Global Optimization, vol. 31, no. 3, pp. 371-381, 2005.

[22] M. Aít Mansour, J. Lahrache, and N. Ziane, "Weak approximate solutions to quasi-variational inequalities: application to social Nash equilibria," Applied Set-Valued Analysis and Optimization, vol. 3, pp. 149-164, 2021.

[23] L. Liu, S. Y. Cho, and J. C. Yao, "Convergence analysis of an inertial Tseng's extragradient algorithm for solving pseudomonotone variational inequalities and applications," Journal of Nonlinear and Variational Analysis, vol. 5, pp. 627-644, 2021. 